\title{
Areas of Polygons Inscribed in a Circle
}

\author{
D. P. Robbins
}

Center for Communications Research, Institute for Defense Analyses, Princeton, NJ 08540, USA

robbins@ccr-p.ida.org

\begin{abstract}
Heron of Alexandria showed that the area $K$ of a triangle with sides $a$, $b$, and $c$ is given by

$$
K=\sqrt{s(s-a)(s-b)(s-c)},
$$

where $s$ is the semiperimeter $(a+b+c) / 2$. Brahmagupta gave a generalization to quadrilaterals inscribed in a circle. In this paper we derive formulas giving the areas of a pentagon or hexagon inscribed in a circle in terms of their side lengths. While the pentagon and hexagon formulas are complicated, we show that each can be written in a surprisingly compact form related to the formula for the discriminant of a cubic polynomial in one variable.
\end{abstract}

\section{Introduction}

Since a triangle is determined by the lengths, $a, b, c$ of its three sides, the area $K$ of the triangle is determined by these three lengths. The well-known formula

$$
K=\sqrt{s(s-a)(s-b)(s-c)}
$$

where $s$ is the semiperimeter $(a+b+c) / 2$, makes this dependence explicit. (This formula is usually ascribed to Heron of Alexandria, $c$. 60 B.C., although some attribute it to Archimedes.)

For polygons of more than three sides, the lengths of the sides do not determine the polygon or its area. However, if we impose the condition that the polygon be convex and cyclic (i.e., inscribed in a circle), then the area of the polygon is uniquely determined. Moreover, it is a symmetric function of the sides. The symmetry can be seen by regarding the polygon as the union of isosceles triangles each bounded 
by two radii and an edge of the polygon. From this point of view changing the order of the sides leaves the area unaffected. Given positive real numbers $a_{1}, \ldots, a_{n}$, a convex $n$-gon can be constructed with the $a_{j}$ 's as the lengths of the sides provided that the largest $a_{j}$ is smaller than the sum of the remaining ones. In this case it is also possible to construct a convex cyclic $n$-gon with the same sides (this is not quite so easy to establish as might be imagined) and this cyclic $n$-gon has the largest area of all $n$-gons with the given side lengths. We refer the reader to pp. 56-60 of [1] for the interesting discussion which brought this problem to our attention.

There is a formula analogous to (1.1), given by Brahmagupta in the seventh century, for the area $K$ of a cyclic quadrilateral whose four sides have lengths $a$, $b, c$, and $d$. It is

$$
K=\sqrt{(s-a)(s-b)(s-c)(s-d)}
$$

where again $s$ is the semiperimeter $(a+b+c+d) / 2$. The proof of this formula is now a fairly routine exercise and is given, for example, in [1].

Curiously this seems to be the end of the line. Apparently no one has derived formulas giving the area of cyclic polygons with more than four sides. In this paper we derive such formulas for pentagons and hexagons and make some conjectures for polygons of more than six sides. While the pentagon and hexagon formulas are complicated, we shall see that it is possible to write them in a surprisingly compact form.

The condition of convexity is algebraically somewhat unnatural, so we also consider the following slightly modified problem. Given positive real numbers $a_{1}, \ldots, a_{n}$, we seek the areas of all $n$-gons, convex or not, whose side lengths are $a_{1}, \ldots, a_{n}$ and whose vertices lie on a circle. Here we take as the definition of the area of a polygon in the Cartesian plane whose vertices are, in order, $\left(x_{1}, y_{1}\right), \ldots,\left(x_{n}, y_{n}\right)$, the expression

$$
\frac{1}{2}\left(\left|\begin{array}{ll}
x_{1} & y_{1} \\
x_{2} & y_{2}
\end{array}\right|+\left|\begin{array}{ll}
x_{2} & y_{2} \\
x_{3} & y_{3}
\end{array}\right|+\cdots+\left|\begin{array}{ll}
x_{n} & y_{n} \\
x_{1} & y_{1}
\end{array}\right|\right)
$$

Defined this way the area is the sum of the areas of the components into which the polygon divides the plane, with each component weighted by winding number. Its sign depends on the direction in which the vertices of the polygon are traversed, but we shall see that all our formulas, like those of Heron and Brahmagupta, involve only the square of the area.

Heron's formula can be restated

$$
16 K^{2}=2 a^{2} b^{2}+2 a^{2} c^{2}+2 b^{2} c^{2}-a^{4}-b^{4}-c^{4},
$$

so that $16 K^{2}$ is a polynomial with integer coefficients in the squares of the sides of the triangle. 
Brahmagupta's formula is

$$
16 K^{2}=2 a^{2} b^{2}+\cdots+2 c^{2} d^{2}-a^{4}-b^{4}-c^{4}-d^{4}+8 a b c d,
$$

so that $16 K^{2}$ is a polynomial in the side lengths in which the exponents of each term are either all even or all odd.

If in Brahmagupta's formula we replace any single side length by its negative, we obtain the formula

$$
16 K^{2}=2 a^{2} b^{2}+\cdots+2 c^{2} d^{2}-a^{4}-b^{4}-c^{4}-d^{4}-8 a b c d
$$

It has a natural geometric interpretation, giving the area, in the sense described above, of a nonconvex quadrilateral inscribed in a circle.

\section{Areas of Cyclic Polygons in General}

In this section we study cyclic polygons of an arbitrary number of sides. The sequence of integers $\Delta_{1}, \Delta_{2}, \Delta_{3}, \ldots=1,7,38,187,874, \ldots$ defined by

$$
\Delta_{m}=\sum_{k=0}^{m-1}(m-k)\left(\begin{array}{c}
2 m+1 \\
k
\end{array}\right)=\frac{1}{2}\left[(2 m+1)\left(\begin{array}{c}
2 m \\
m
\end{array}\right)-2^{2 m}\right]
$$

is important in what follows. Here is the strongest result which we are able to prove for cyclic polygons.

Theorem 1. Given $n$ a unique (up to sign) irreducible homogeneous (regarding the first argument as having degree 4 and the remaining arguments as having degree 2) polynomial $f$ exists with integer coefficients such that whenever $\left(a_{1}, \ldots, a_{n}\right)$ are the side lengths of a cyclic n-gon and $K$ is its area, then

$$
f\left(16 K^{2}, a_{1}^{2}, \ldots, a_{n}^{2}\right)=0
$$

This polynomial is symmetric in the $a_{j}^{2}$. If $n=2 m+1$, the degree of this polynomial in its first variable, $16 K^{2}$, is at least $\Delta_{m}$. If $n=2 m+2$, the degree of this polynomial in $16 K^{2}$ is at least $2 \Delta_{m}$.

Additional Conjectures. We believe that the stated degree bounds are really equalities and that the polynomial, with the proper choice of sign, is always a monic polynomial in its first argument. Furthermore, we believe that when $n=2 m+2$ is even, the polynomial factors as a product of two monic polynomials of degree $\Delta_{m}$, whose coefficients are polynomials in side lengths (but not their squares). We are not able to prove these conjectures except in the cases that $n \leq 6$. 
Proof of Theorem 1. First we show that such polynomials exist. We may assume that the polygon has its vertices on a circle centered at the origin in the complex plane. Suppose that these vertices are in order $v_{1}, \ldots, v_{n}$ and that the radius of the circle is $R$. Also let $v_{n+1}=v_{1}$ and define the quotients $q_{j}=v_{j+1} / v_{j}, j=1, \ldots, n$. Then, letting $a_{j}$ be the distance from $v_{j}$ to $v_{j+1}$, we have

$$
a_{j}^{2}=\left|v_{j+1}-v_{j}\right|^{2}=R^{2}\left(v_{j+1}-v_{j}\right)\left(1 / v_{j+1}-1 / v_{j}\right)=R^{2}\left(2-q_{j}-q_{j}^{-1}\right) .
$$

The area of the triangle with vertices $0, v_{j}$, and $v_{j+1}$ is given by

$$
\frac{1}{2} \operatorname{Im}\left(\bar{v}_{j} v_{j+1}\right)=(1 / 4 i) R^{2}\left(v_{j+1} / v_{j}-v_{j} / v_{j+1}\right) .
$$

Hence we have

$$
-16 K^{2}=R^{4}\left(v_{1} / v_{2}-v_{2} / v_{1}+\cdots\right)^{2}=R^{4}\left(q_{1}+\cdots+q_{n}-q_{1}^{-1}-\cdots-q_{n}^{-1}\right)^{2} .
$$

It follows that the quantities $16 K^{2} / a_{j}^{4}$ are $n$ rational functions of the $q_{j}^{\text {'s. }}$

Now observe that the $q_{j}$ 's satisfy the relation $q_{1} \cdots q_{n}=1$ and are therefore algebraically dependent over the rational numbers. Hence the functions $16 K^{2} / a_{j}^{4}$, which are rational functions of the $q_{j}$ 's, must themselves be algebraically dependent over the rationals. Thus a polynomial $f$ such that (2.1) holds exists. (The polynomial whose existence we have established involves only fourth powers of the side lengths and so is a fortiori of the correct form.) Now regard $f$ as an element of the unique factorization domain of polynomials in $16 K^{2}$ and the $a_{j}^{2}$ 's with integer coefficients and factor $f$ into irreducible polynomials $f=f_{1} \cdots f_{s}$ in this domain. The factors $f_{j}$ will have the same homogeneity property as $f$. We show that (2.1) holds for at least one of these irreducibles. If we substitute in the equation $f=0$ the relations (2.2) and (2.3) expressing $16 K^{2}$ and the $a_{j}^{2}$ in terms of the $v_{j}$ 's, we obtain a power of $R$ times a Laurent polynomial in the $v_{j}$ 's. This Laurent polynomial vanishes identically.

Because of the homogeneity of the irreducible factors $f_{j}$, when we make the same substitution in the $f_{j}$ 's we again obtain powers of $R$ times certain Laurent polynomials in the $v_{j}$ 's. However, the set of all Laurent polynomials is an integral domain. Hence we can deduce that the polynomial obtained by substituting in one of the irreducible factors, say $f_{1}$, is zero. It follows, regarding $16 K^{2}$ and $a_{j}^{2}$ as polynomials in the $v_{j}$ 's, that

$$
f_{1}\left(16 K^{2}, a_{1}^{2}, \ldots, a_{n}^{2}\right)=0
$$

holds for all nonzero $v_{1}, \ldots, v_{n}$. In particular, when the $v_{j}$ 's are on a circle centered at the origin, we can interpret the first argument as the area and the last $n$ arguments as the squared lengths of the sides. Therefore $f_{1}$ is an irreducible polynomial satisfying (2.1).

Suppose that $f_{1}$ and $f_{2}$ are two irreducible polynomials with integer coefficients such that (2.1) holds for convex cyclic $n$-gons. Then, taking the resultant with 
respect to the first argument, $16 K^{2}$, we can eliminate $16 K^{2}$ from these equations obtaining an algebraic relation that held among the $a_{j}^{2}$ 's of cyclic polygons. In particular, this relation must hold for the open set of all $n$-tuples of positive real numbers $a_{1}, \ldots, a_{n}$ for which it is possible to construct a convex cyclic $n$-gon. It follows that the resultant is identically zero and therefore the polynomials have a common zero in their first variable. However, if irreducible polynomials share a common zero, they must have all their roots in common. It then easily follows that the polynomials are the same except for sign. Because the polynomial $f$ is uniquely determined by the areas and side lengths of convex cyclic $n$-gons, and the areas of such $n$-gons are symmetric functions of the side lengths, it follows that the coefficients of $f$ are symmetric polynomials in the side lengths.

Finally we prove the lower bound on the degree. To do this it suffices to show that, for each $n$, there are sets of sides for which there are at least the given number of distinct areas. Here our argument is quite informal.

Suppose that $n=2 m+1$ is odd. It suffices to take the side lengths to be distinct but nearly equal. This having been done, imagine a circle of variable radius and try to inscribe a polygon with sides of the given lengths in the circle by picking an arbitrary starting point and laying out the edges, one at a time, with the given lengths. When the radius is too large, we will not reach the starting point when we have used up all the sides. As we decrease the radius there will come a time when we return exactly to our starting point. The resulting polygon will be nearly the regular polygon with $n$ sides. If we continue to decrease the radius, we will overshoot the starting point starting to go around the circle again. When the radius has decreased enough, we will go around the circle exactly twice, creating a star-shaped polygon. We can continue this way finding radii requiring more trips around the circle yielding stars with sharper points. However, no edge can go as much as half way around the circle so that the maximum number of times we can go around is $m$. This is where our first $m$ circles come from and the squared areas of the corresponding polygons will be generically distinct.

There are other solutions. These arise as follows. We have so far assumed (implicitly) that, as we lay out all the sides around the circle, we are always proceeding in the same direction. However, this is not necessary. We can lay down one of the sides in the opposite direction. Then we get a solution which looks something like a $(2 m-1)$-gon because the backward edge almost coincides with the preceding and following edges. Here we have $2 m+1$ choices for the backward edge and for each of these choices we can still go around the circle $m-1$ times. In general each choice will require a different radius and yield a different area.

This explains the term $(m-1)\left(\begin{array}{c}2 m+1 \\ 1\end{array}\right)$ in the formula defining $\Delta_{m}$. Subsequent terms are explained by selecting more, up to $m-1$, of the sides to go backward. This completes our proof for the case of $n$ odd.

Next suppose $n=2 m+2$ is even. Here we take $2 m+1$ of the sides to be nearly equal and the last side to be very small. Then we can construct $\Delta_{m}$ solutions with the $2 m+1$ sides in the same orientation as above and the very small side proceeding in the same direction as the majority. We can also construct other $\Delta_{m}$ 

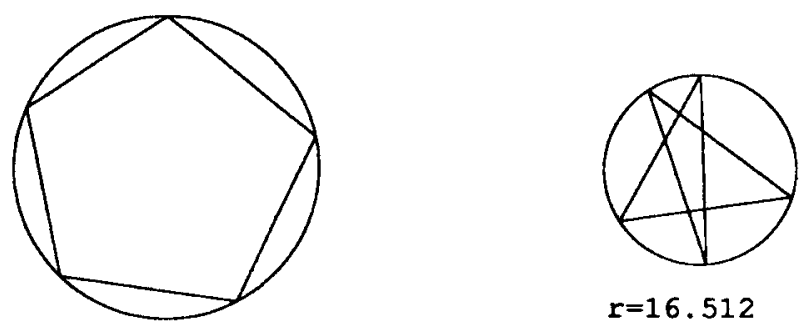

$r=16.512$

$r=26.385$

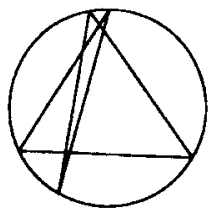

$r=17.026$

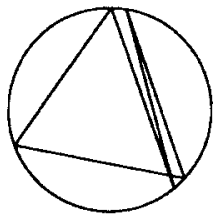

$r=17.595$

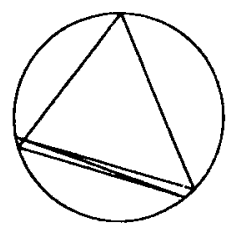

$r=17.991$

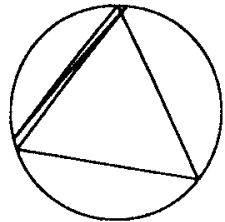

$r=18.335$

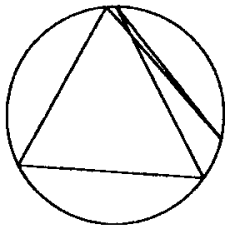

$r=18.651$

Fig. 1. The seven cyclic pentagons.

solutions with the very small side proceeding in the opposite direction. This completes the (quite informal) proof.

We denote by $\alpha_{n}$ the polynomial constructed in Theorem 1 .

Figure 1 illustrates the seven cyclic pentagons with side lengths $29,30,31,32$, and 33.

\section{Area of a Cyclic Pentagon}

In this section we give an explicit construction of the polynomial $\alpha_{5}$ from Theorem 1.

While it is not a logical necessity, we digress to describe how this polynomial was discovered. We suspected from the outset, by analogy with the case of triangles and cyclic quadrilaterals, that the polynomial would be monic with integer coefficients. We then also gained evidence for this conjecture by choosing pentagons with nearly equal integer sides so that the seven distinct solutions, described 
in the proof of Theorem 1, existed and, for each solution, we computed $16 K^{2}$ using high-precision arithmetic. We then formed the monic polynomial whose roots were the seven quantities so obtained and observed that, with sufficient precision, the coefficients of this polynomial were nearly integers, as predicted.

An elaboration of the previous confirmation method also allows for computation of the correct formula. We decided to express the coefficients of the powers of $16 K^{2}$ in terms of the elementary symmetric functions

$$
\begin{aligned}
& \sigma_{1}=a_{1}^{2}+\cdots+a_{5}^{2}, \\
& \sigma_{2}=a_{1}^{2} a_{2}^{2}+\cdots+a_{4}^{2} a_{5}^{2},
\end{aligned}
$$

of $a_{1}^{2}, \ldots, a_{5}^{2}$. Thus, taking into account the homogeneity properties of the polynomial $f$ we were seeking, we expected $f$ to have the form

$$
f=u^{7}+\left(c_{1} \sigma_{1}^{2}+c_{2} \sigma_{2}\right) u^{6}+\left(c_{3} \sigma_{1}^{4}+\cdots\right) u^{5}+\cdots
$$

where $u=16 K^{2}$ for brevity and $c_{1}, c_{2}, c_{3}, \ldots$ were certain integer constants to be determined. Since for a given pentagon we can compute this polynomial exactly (rounding the near integer coefficients to the nearest integer) and we can compute the $\sigma_{j}$ 's, each such example gives us linear equations satisfied by the $c_{j}$ 's, one equation for each power of $u$. A simple enumeration shows that only 70 unknown $c_{j}$ 's are involved in the most complicated coefficient which is the constant term. Thus with 70 examples (and a little luck so that the resulting systems of equations are nonsingular) we can solve for the unknown coefficients.

This is how we found the formula in the first place. An additional check on the correctness of our calculations, which were, after all, based on approximate arithmetic, was that the computed $c_{j}$ 's were integers. More striking, however, was the observation that all the $c_{j}$ 's were quite round numbers involving only small primes in their prime factorizations. This suggested that the polynomial possessed some additional structure.

By examining the polynomial carefully we then observed, with the help of the computer program Mathematica, that it could indeed be written in a simpler form. Specifically, we found that the equation for $u$ (still an abbreviation for $16 K^{2}$ ) could be written as

$$
\alpha_{5}=-t_{3}^{2} t_{4}^{2}-u t_{4}^{3}+16 t_{3}^{3} t_{5}+18 u t_{3} t_{4} t_{5}+27 u^{2} t_{5}^{2}=0
$$

where

$$
\begin{aligned}
& t_{2}=u-4 \sigma_{2}+\sigma_{1}^{2}, \\
& t_{3}=8 \sigma_{3}+\sigma_{1} t_{2}, \\
& t_{4}=-64 \sigma_{4}+t_{2}^{2}, \\
& t_{5}=128 \sigma_{5} .
\end{aligned}
$$


Note that the formula yields a monic polynomial of degree 7 for $u$ whose coefficients are polynomials in the squares of the lengths of the sides. Having discovered the formula (3.1), we had abandoned it not understanding its significance. Some time later Bradley Brock pointed out the extremely interesting fact that the left-hand side of (3.2) resembled the discriminant of a cubic. Indeed it is precisely $1 /\left(4 u^{2}\right)$ times the discriminant with respect to $z$ of the cubic polynomial

$$
z^{3}+2 t_{3} z^{2}-u t_{4} z+2 u^{2} t_{5}
$$

Why this should be the case is still a mystery. However, the observation leads to a relatively simple verification of the correctness of the formula which could be carried out entirely by hand.

Recall the $q_{j}$ 's of Section 2 and the relations

$$
a_{j}^{2}=R^{2}\left(2-q_{j}-q_{j}^{-1}\right)
$$

and

$$
16 K^{2}=-R^{4}\left(q_{1}+\cdots+q_{5}-q_{1}^{-1}-\cdots-q_{5}^{-1}\right)^{2}
$$

From (3.2) we can, in principle, express the elementary symmetric functions of the five $a_{j}^{2}$ in terms of the elementary symmetric functions $\tau_{1}, \ldots, \tau_{5}$ of the $q_{j}$ 's. Here is one way to do it in practice. Note that $\tau_{5}=1$. Let

$$
f(x)=\prod_{j=1}^{5}\left(x-q_{j}\right)=x^{5}-\tau_{1} x^{4}+\tau_{2} x^{3}-\tau_{3} x^{2}+\tau_{4} x-1
$$

and

$$
g(x)=x^{2}+\left(y / R^{2}-2\right) x+1
$$

where $y$ is an indeterminate. Then $f(x)=0$ and $g(x)=0$ have a common solution exactly when $q_{j}^{2}+\left(y / R^{2}-2\right) q_{j}+1=0$ or $y=a_{j}^{2}$ for some $j$. Thus if we express the resultant of $f$ and $g$ with respect to $x$ in powers of $y$, then the coefficient of $y^{5-j}$ is $(-1)^{j}$ times the expression for $\sigma_{j}$ in terms of the $\tau$ 's. This way we find that

$$
\begin{aligned}
\sigma_{1}= & R^{2}\left(10-\tau_{1}-\tau_{4}\right), \\
\sigma_{2}= & R^{4}\left(35-8 \tau_{1}+\tau_{2}+\tau_{3}-8 \tau_{4}+\tau_{1} \tau_{4}\right) \\
\sigma_{3}= & R^{6}\left(50-20 \tau_{1}+5 \tau_{2}+5 \tau_{3}-20 \tau_{4}-\tau_{1} \tau_{3}-\tau_{2} \tau_{4}+6 \tau_{1} \tau_{4}\right), \\
\sigma_{4}= & R^{8}\left(25-15 \tau_{1}+5 \tau_{2}+5 \tau_{3}-15 \tau_{4}+\tau_{1} \tau_{2}+\tau_{2} \tau_{3}+\tau_{3} \tau_{4}-4 \tau_{1} \tau_{3}\right. \\
& \left.\quad-4 \tau_{2} \tau_{4}+9 \tau_{1} \tau_{4}\right), \\
\sigma_{5}= & -R^{10}\left(\tau_{1}-\tau_{2}+\tau_{3}-\tau_{4}\right)^{2} .
\end{aligned}
$$


Also

$$
16 K^{2}=-R^{4}\left(\tau_{1}-\tau_{4}\right)^{2}
$$

Next we express the $t$ 's in terms of the $\tau$ 's and we find that

$$
\begin{aligned}
& t_{2}=4 R^{4}\left(-10+3 \tau_{1}-\tau_{2}-\tau_{3}+3 \tau_{4}\right) \\
& t_{3}=-4 R^{6}\left(3 \tau_{1}-\tau_{2}+\tau_{3}-3 \tau_{4}\right)\left(\tau_{1}-\tau_{4}\right) \\
& t_{4}=16 R^{8}\left(9 \tau_{1}-\tau_{2}+\tau_{3}-9 \tau_{4}\right)\left(-\tau_{1}+\tau_{2}-\tau_{3}+\tau_{4}\right) \\
& t_{5}=-128 R^{10}\left(\tau_{1}-\tau_{2}+\tau_{3}-\tau_{4}\right)^{2}
\end{aligned}
$$

Note that each of $t_{3}, t_{4}$, and $t_{5}$ factor as a product of two linear functions in the $\tau$ 's. This may explain their significance. It can now be easily verified that

$$
\begin{aligned}
z^{3}+2 t_{3} z^{2} & -u t_{4} z+2 u^{2} t_{5} \\
& =\left(z-16 R^{6}\left(\tau_{1}-\tau_{4}\right)^{2}\right)\left(z-4 R^{6}\left(\tau_{1}-\tau_{2}+\tau_{3}-\tau_{4}\right)\left(\tau_{1}-\tau_{4}\right)\right)^{2} .
\end{aligned}
$$

Since the cubic has a double root, we may conclude that its discriminant is 0 . Thus we have proved the formula for the pentagon area.

\section{Area of the Hexagon}

In this section we derive the polynomial $\alpha_{6}$ giving the areas of cyclic hexagons in terms of their side lengths $a_{1}, \ldots, a_{6}$. We originally found this formula in much the same way as we did that of the pentagon.

However, we made an additional guess. Theorem 1 asserts the existence of a degree 14 polynomial in $16 K^{2}$ whose coefficients are polynomials in the squared sides lengths. Because of the relatively high degree it would be difficult to carry out the calculations directly using the method for the pentagon. However, by analogy with Brahmagupta's formula, we expected that the polynomial $\alpha_{6}$ would factor $\beta_{6} \beta_{6}^{*}$ where $\beta_{6}$ and $\beta_{6}^{*}$ are polynomials of degree 7 in $u=16 K^{2}$ and in which the coefficients of the powers of $u$ are symmetric polynomials, each with the property that the exponents of the $a_{j}$ 's in every term are either all odd or all even. Stated in terms of symmetric functions each coefficient would be a polynomial with integer coefficients in the first five elementary symmetric functions $\sigma_{1}, \ldots, \sigma_{5}$ of the $a_{j}^{2}$ 's and the function $\sigma_{6}^{\prime}=a_{1} \cdots a_{6}$.

To carry out the rest of the plan we needed to know how to partition the 14 cyclic hexagons that could be constructed from a suitable set of six side lengths into two 7-element subsets, one subset whose areas are the roots of $\beta_{6}$ and the other whose roots are the areas of $\beta_{6}^{*}$. We shall see that the following construction gives the correct partition. 
Let us assume that the vertices of our convex hexagon are complex numbers $v_{1}, \ldots, v_{6}$ on a circle centered at the origin in the complex plane and that $q_{j}=v_{j+1} / v_{j}$ are the vertex quotients. Observe that the quantity

$$
p=\left(1-q_{1}\right)\left(1-q_{2}\right) \cdots\left(1-q_{6}\right)
$$

is always real. Indeed, since the $q_{j}$ 's have absolute value one,

$$
\begin{aligned}
\bar{p} & =\left(1-q_{1}^{-1}\right) \cdots\left(1-q_{6}^{-1}\right) \\
& =q_{1}^{-1}\left(q_{1}-1\right) \cdots q_{6}^{-1}\left(q_{6}-1\right) \\
& =\left(q_{1} \cdots q_{6}\right)^{-1} p=p .
\end{aligned}
$$

Thus hexagons fall into two categories according to whether $p$ is positive or negative. In the construction of Theorem 1 it is seen that precisely seven of the hexagons have positive values of $p$ and that seven have negative values, the convex hexagon falling into the positive category. We guessed that this was the correct partition in order to compute our formula.

We remark that there is a simple geometric interpretation of the sign of $p$. Starting with an arbitrary hexagon, let the vertices of the hexagon move continuously around the circle, in such a way that no three consecutive vertices ever coincide, until the hexagon becomes convex. At certain times in the process two consecutive vertices will cross. At these times the product $p$ changes sign. Thus $p$ is positive exactly when the number of crossings required is even so that it makes sense to call the sign of $p$ the crossing parity.

Assuming all these guesses were correct, we used the same undetermined coefficients method as was used for the pentagon to determine the correct formula for $\beta_{6}$ (which we took to be the factor corresponding to the hexagons with even crossing parity). The calculation is slightly more complicated because now the most complicated system of equations has 134 unknowns.

Again, once the formula was produced, it was apparent that it possessed some additional structure much as did the pentagon formula. Indeed, the formula was the same as (3.2) except that we had to modify the definitions of the $t$ 's. We found that

$$
\beta_{6}=-t_{3}^{2} t_{4}^{2}-u t_{4}^{3}+16 t_{3}^{3} t_{5}+18 u t_{3} t_{4} t_{5}+27 u^{2} t_{5}^{2},
$$

where now

$$
\begin{aligned}
& t_{2}=u-4 \sigma_{2}+\sigma_{1}^{2}, \\
& t_{3}=8 \sigma_{3}+\sigma_{1} t_{2}-16 \sigma_{6}^{\prime}, \\
& t_{4}=t_{2}^{2}-64 \sigma_{4}+64 \sigma_{1} \sigma_{6}^{\prime}, \\
& t_{5}=128 \sigma_{5}+32 t_{2} \sigma_{6}^{\prime} .
\end{aligned}
$$


Again it is not difficult, once the formula is found, to verify it directly. Let $\tau_{1}, \ldots, \tau_{6}$ be the elementary symmetric functions of the $q_{j}$ 's. We have $\tau_{6}=1$. Also

$$
16 K^{2}=-R^{4}\left(q_{1}+\cdots+q_{6}-q_{1}^{-1}-\cdots-q_{6}^{-1}\right)^{2}=-R^{4}\left(\tau_{1}-\tau_{5}\right)^{2}
$$

and

$$
a_{j}^{2}=R^{2}\left(2-q_{j}-q_{j}^{-1}\right)
$$

We express the elementary symmetric functions of the $a_{j}^{2}$ 's in terms of the $\tau$ 's, using the same method as we used for the pentagon. This yields

$$
\begin{aligned}
\sigma_{1}= & R^{2}\left(12-\tau_{1}-\tau_{5}\right), \\
\sigma_{2}= & R^{4}\left(54-10 \tau_{1}+\tau_{2}+\tau_{4}-10 \tau_{5}+\tau_{1} \tau_{5}\right), \\
\sigma_{3}= & R^{6}\left(112-35 \tau_{1}+8 \tau_{2}-2 \tau_{3}+8 \tau_{4}-35 \tau_{5}-\tau_{1} \tau_{4}-\tau_{2} \tau_{5}+8 \tau_{1} \tau_{5}\right), \\
\sigma_{4}= & R^{8}\left(105-50 \tau_{1}+21 \tau_{2}-12 \tau_{3}+\cdots+\tau_{1} \tau_{3}+\cdots-6 \tau_{1} \tau_{4}+\cdots+20 \tau_{1} \tau_{5}\right), \\
\sigma_{5}= & R^{10}\left(36-26 \tau_{1}+20 \tau_{2}-18 \tau_{3}+\cdots-\tau_{1} \tau_{2}-\cdots+4 \tau_{1} \tau_{3}\right. \\
& \left.\quad+\cdots-9 \tau_{1} \tau_{4}-\cdots+16 \tau_{1} \tau_{5}\right) .
\end{aligned}
$$

We can also express $\sigma_{6}^{\prime}$ in terms of the $\tau$ 's. For those hexagons for which $p>0$, we have

$$
\begin{aligned}
\sigma_{6}^{\prime} & =\left|v_{1}-v_{2}\right|\left|v_{2}-v_{3}\right| \cdots\left|v_{6}-v_{1}\right| \\
& =\left|v_{1} \cdots v_{6}\right||p|=R^{6}|p|=R^{6} p \\
& =R^{6}\left(1-q_{1}\right) \cdots\left(1-q_{6}\right) \\
& =R^{6}\left(1-\tau_{1}+\tau_{2}-\tau_{3}+\tau_{4}-\tau_{5}+1\right) .
\end{aligned}
$$

Next we express the $t$ 's in terms of the $\tau$ 's. We obtain

$$
\begin{aligned}
& t_{2}=4 R^{4}\left(-18+4 \tau_{1}-\tau_{2}-\tau_{4}+4 \tau_{5}\right) \\
& t_{3}=-4 R^{6}\left(4 \tau_{1}-\tau_{2}+\tau_{4}-4 \tau_{5}\right)\left(\tau_{1}-\tau_{5}\right) \\
& t_{4}=16 R^{8}\left(10 \tau_{1}-\tau_{2}+\tau_{4}-10 \tau_{5}\right)\left(2 \tau_{1}-\tau_{2}+\tau_{4}-2 \tau_{5}\right), \\
& t_{5}=-128 R^{10}\left(2 \tau_{1}-\tau_{2}+\tau_{4}-2 \tau_{5}\right)^{2}
\end{aligned}
$$

Again note that each of $t_{3}, t_{4}$, and $t_{5}$ factor as a product of two linear functions in the $\tau$ 's. We also find that

$$
\begin{aligned}
& z^{3}+2 t_{3} z^{2}-u t_{4} z+2 u^{2} t_{5} \\
& \quad=\left(z-16 R^{6}\left(\tau_{1}-\tau_{5}\right)^{2}\right)\left(z-4 R^{6}\left(2 \tau_{1}-\tau_{2}+\tau_{4}-2 \tau_{5}\right)\left(\tau_{1}-\tau_{5}\right)\right)^{2} .
\end{aligned}
$$


Since the cubic in $z$ has a double root, we have verified the formula for the hexagon.

The polynomial $\beta_{6}^{*}$ for cyclic hexagons of odd crossing parity can be obtained from $\beta_{6}$ by replacing $\sigma_{6}^{\prime}$ by its negative. We now see that $\alpha_{6}=\beta_{6} \beta_{6}^{*}$.

In principle the same method could be used for the polygons of more sides. We have in fact verified in a few examples that if we take seven nearly equal integer side lengths, then the 38 values of $16 K^{2}$ do indeed satisfy a monic polynomial with integer coefficients. However, it would be a considerable computational problem to carry out the undetermined coefficients method since the largest system of equations would involve 143,307 undetermined coefficients. Even if we were able to compute the polynomial $\alpha_{7}$ this way, it would obviously be a substantial problem to manipulate it into a more compact form. Thus it seems best to guess the compact form first should one exist.

\section{The Circumradius}

The circumradius of a cyclic polygon is also a symmetric function of the side lengths and it is possible to study it in a manner analogous to our treatment of the area. We have done this and derived the formulas for the circumradius for the case of the pentagon and hexagon. Here we have a somewhat simpler inductive method available. We can derive a formula for the $n$-gon by cutting it along a diagonal into a cyclic quadrilateral and an $(n-2)$-gon. Then we use the previously derived formulas for the circumradius of the $(n-2)$-gon and the cyclic quadrilateral. These formulas involve two unknowns, the circumradius and the length of the chosen diagonal. Taking the resultant of these two formulas with respect to the diagonal, we eliminate the diagonal and construct a polynomial satisfied by the circumradius of the $n$-gon. This seems always to give a power of $R$ times the appropriate minimum polynomial for $R^{2}$, but we are unable to prove this. Sillke [2] has derived the formula for the cyclic pentagon by similar methods.

Although the coefficients in the resulting formulas for both the pentagon and the hexagon again factor into small primes, we have been unable to put either formula into a sensible compact form. However, based on our experience we can give some conjectures about these formulas. Some facts relating to the degrees of the minimum polynomials can be proved just as for the area formula.

We begin with the circumradius of the $(2 m+1)$-gon.

Conjecture 1. Let $m$ be a positive integer. Then a unique (up to sign) homogeneous polynomial $\rho_{2 m+1}$ of $2 m+2$ variables with integer coefficients, of degree $\Delta_{m}$ in its first variable and of overall degree $(2 m+1)\left(\begin{array}{c}2 m \\ m\end{array}\right)$, exists such that whenever a polygon with $2 m+1$ sides of lengths $a_{1}, a_{2}, \ldots, a_{2 m+1}$ is inscribed in a circle of radius $R$, then

$$
\rho_{2 m+1}\left(R^{2}, a_{1}, a_{2}, \ldots, a_{2 m+1}\right)=0 .
$$


This polynomial is irreducible and symmetric in the $a_{j}$ 's and involves only even powers of them. The ambiguous sign can be chosen so that the coefficient of $R^{2 \Delta_{m}}$ is the product of $2^{2 m}$ factors of the form

$$
\pm a_{1} \pm a_{2} \cdots \pm a_{2 m+1}
$$

where the allowed sign patterns are those with more plus signs than minus signs and the term independent of $R$ is

$$
(-1)^{\Delta_{m}}\left(\prod_{j=1}^{2 m+1} a_{j}\right)^{\left(\begin{array}{c}
2 m \\
m
\end{array}\right)}
$$

(It may be easily proved that $\Delta_{m}$ is odd exactly when $m$ is a power of 2.)

The situation is somewhat different for polygons with an even number of sides. Again we distinguish two types according to the crossing parity as described in the last section. Here are the conjectures for $(2 m+2)$-gons.

Conjecture 2. Let $m$ be a positive integer. Then a unique (up to sign) homogeneous polynomial $\rho_{2 m+2}$ of $2 m+3$ variables with integer coefficients, of degree $\Delta_{m}$ in its first variable and overall degree $(2 m+1)\left(\begin{array}{c}2 m \\ m\end{array}\right)$, exists such that whenever a polygon with $2 m+2$ sides of lengths $a_{1}, a_{2}, \ldots, a_{2 m+2}$ is inscribed in a circle of radius $R$ and has even crossing number, then

$$
\rho_{2 m+2}\left(R^{2}, a_{1}, a_{2}, \ldots, a_{2 m+2}\right)=0 .
$$

This polynomial is symmetric in the $a_{j}$ 's, with each term consisting either entirely of even powers of the $a_{j}$ 's or entirely of odd powers. The ambiguous sign can be chosen so that the coefficient of $R^{2 \Delta_{m}}$ is the product of $2^{2 m}$ factors of the form

$$
\pm a_{1} \pm a_{2} \cdots \pm a_{2 m+2}
$$

where the allowed sign patterns have more plus signs than minus signs and the number of plus signs has the same parity as $m$, and the term independent of $R$ is

$$
(-1)^{\Delta_{m}} \prod_{S, T}\left(\prod_{j \in S} a_{j}+\prod_{j \in T} a_{j}\right),
$$

where, in the first product, $S$ and $T$ run over all disjoint pairs of $(m+1)$-element subsets of $\{1, \ldots, 2 m+2\}$ with $1 \in S$.

In the cases $m=1$ of Conjectures 1 and 2, the formulas for the circumradius are completely specified and correspond to well-known formulas for the circum- 
radius of a triangle and a cyclic quadrilateral. We have also verified the correctness of our conjectures for pentagons and hexagons and obtained some evidence for their correctness in the case of heptagons.

For $(2 m+2)$-gons with odd crossing number, we can expect the formulas of Conjecture 2 to hold if we replace one of the sides of the polygon with its negative.

\section{Acknowledgments}

A number of colleagues have made helpful suggestions, some of which have been incorporated in the exposition. The author would like particularly to acknowledge the contributions of bradley Brock, Russell Kulsrud, David Lieberman, and James Maiorana.

\section{References}

1. H. S. M. Coxeter and S. L. Greitzer, Geometry Revisited, The Mathematical Association of America, Washington, DC, 1967.

2. Torsten Sillke, Private communication.

Received March 3, 1993, and in revised form October 14, 1993. 